\title{
Dringlichkeit vs. Nutzen bei der Allokation von Organen
}

\author{
Aus dem Transplantationsgesetz und den darin festgelegten Zuteilungskriterien \\ ergeben sich zahlreiche Konsequenzen. Dass medizinisch dringliche Patienten bei \\ der Zuteilung von Organen prioritär behandelt werden, birgt auch Probleme. Es gibt \\ alternative Möglichkeiten zur Allokation der spärlich vorhandenen Spenderorgane.
}

Marc Marthaler,

Julius Weiss, Franz F. Immer

Swisstransplant, Schweizerische Nationale Stiftung für Organspende und Transplantation
Korrespondenz:

PD Dr. med. Franz F. Immer

FMH Herz- und thorakale

Gefässchirurgie

Direktor Swisstransplant

Laupenstrasse 37

CH-3008 Bern

Tel. 0313808133

Fax 0313808132

franz.immer@

swisstransplant.org

www.swisstransplant.org

\section{Einleitung}

Auch mehr als zwei Jahre nach Inkrafttreten des Transplantationsgesetzes debattieren Juristen, Mediziner und Ethiker weiterhin über die in der Schweiz geltenden Zuteilungskriterien. Anlass dazu ist in erster Linie die Tatsache, dass nach wie vor - und wohl auch bis auf weiteres - ein Mangel an Spenderorganen herrscht. Bei der Verteilung von nur in begrenzter Anzahl vorhandenen Gütern, die wie im vorliegenden Fall zudem oft über Leben oder Tod von Menschen entscheiden oder wovon zumindest deren Lebensqualität massgeblich abhängt, kommt dem Gesetzgeber eine grosse Verantwortung zu. Grundlage für die Zuteilung von Spenderorganen bildet in der Schweiz Artikel 119a der Bundesverfassung, der besagt, dass der Bund Kriterien für eine gerechte Zuteilung von Organen festlegt. Im Art. 18 des Transplantationsgesetzes werden folgende Kriterien für die Zuteilung genannt:

- die medizinische Dringlichkeit

- der medizinische Nutzen

- die Wartezeit

Die folgenden Ausführungen sind als Übersicht über Konsequenzen und Probleme zu verstehen, die sich aus dem Transplantationsgesetz und den darin festgelegten Zuteilungskriterien ergeben. Schliesslich sollen alternative Möglichkeiten der Allokation spärlich vorhandender medizinischer Ressourcen aufgezeigt werden, die den besprochenen Punkten Rechnung tragen sollen.

\section{Zuteilungspraxis}

In der Schweiz ist durch das Transplantationsgesetz und die Zuteilungsverordnungen ein rigider Rahmen für die Zuteilung von Spenderorganen vorgegeben. Die stärkere Gewichtung der Dringlichkeit gegenüber dem Nutzen zeigt, dass der Gesetzgeber offenbar eine Position vertritt, bei der der Wert von Individuen nicht verhandelbar ist und die dem Überleben von Kandidaten für eine Transplantation das grösste Gewicht verleiht.

Weiter haben aufgrund der patientenorientierten, nationalen Zuteilung die Transplantationszentren sehr wenig Mitspracherecht, wenn es darum geht zu entscheiden, welchem Patienten ein Organ zugeteilt wird. Im Grunde haben die Zentren lediglich das Recht, Organangebote an einen bestimmten Patienten aus medizinischen Gründen abzulehnen.

Unter der Annahme, dass Transplantationszentren generell ein Interesse daran haben, ihre Patienten zu transplantieren (soziale Nähe zu den eigenen Patienten, Prestige der Transplantationsmedizin), hat dieses System weitreichende Konsequenzen: auf der einen Seite führt das System dazu, Patienten tendenziell früh(er) auf die Warteliste aufzunehmen, um Wartezeit zu generieren und auch Patienten zu listen, bei denen die Aussicht auf eine gelingende Transplantation mit einem guten Outcome eher gering ist. Denn statistisch betrachtet liegt es auf der Hand, dass Zentren mit grossen Wartelisten und mit Patienten mit langen Wartezeiten anzahlmässig mehr Organe erhalten werden. Weiter dürfte die Tendenz dazu bestehen, Organangebote wenn immer möglich zu akzeptieren, denn ein Organangebot abzulehnen, kommt einer verpassten Chance gleich, für den Patienten wie auch für das Zentrum.

Die durch das Gesetz geschaffenen «Zwänge» haben vor dem Inkrafttreten des Transplantationsgesetzes im Rahmen der lokalen Zuteilung nicht in der gleichen Weise bestanden. Einige Vorteile der lokalen Zuteilung sind denn auch augenscheinlich: Organe von hausinternen Spendern konnten den eigenen Patienten zugeteilt werden, was eine Zuteilung an die am besten passenden Empfänger ermöglicht hat. Dass die lokale Zuteilung zudem einen positiven Einfluss auf die Motivation der Spenderdetektion der Zentren haben dürfte, scheint unbestreitbar. Wenn Organe hausinterner Spender wenn immer möglich auch den eigenen Patienten zugute kommen, dann stellt dies zweifellos einen Anreiz dar, sich der Spenderarbeit anzunehmen.

\section{Medizinisch}

Wenn begrenzt verfügbare medizinische Ressourcen de lege prioritär an schwer kranke Patienten gehen, und auf den Nutzen abzielende Kriterien erst zum Zug kommen, wenn es keine Patienten mit einer medizi- 
nischen Dringlichkeit gibt, dann bleiben für Patienten, die sich nicht in akuter Lebensgefahr oder zumindest in einer gesundheitlich weniger bedrohlichen Situation befinden, weniger dieser Ressourcen übrig. Herrscht akuter Mangel an diesen Ressourcen, so treten nutzenorientierte Überlegungen bei der Zuteilung stärker in den Hintergrund. auf einzelne Patienten, längerfristig kostensparend, es darf jedoch nicht übersehen werden, dass die oben beschriebenen Folgen der Zuteilung gemäss medizinischer Dringlichkeit insgesamt mit Mehrkosten verbunden sind. Sowohl die Behandlung der «weniger» kranken Patienten, die aufgrund fehlender Dringlichkeit der Transplantation länger auf ein Organ warten

\section{Organe bekommen Patienten, die am dringendsten darauf angewiesen sind. Die übrigen Patienten auf der Warteliste müssen warten, bis sie krank genug sind}

Die Folgen der Zuteilung nach medizinischer Dringlichkeit lässt sich gut an der Zuteilung von Herzen und Lebern illustrieren. Bei den Herzen ist die Dringlichkeit ein diskretes Kriterium, d.h. ein Patient gilt entweder als dringlich (urgent) oder nicht. Bei Lebern gibt es zwar den Urgent-Status ebenfalls, jedoch ist in den allermeisten Fällen der MELD-Score* ausschlaggebend für die Priorität der Patienten bei der Zuteilung; je höher der MELD-Score, desto höher die Priorität des Patienten.

In der Zeit zwischen dem Inkrafttreten des Transplantationsgesetzes am 1. Juli 2007 und dem 1. Januar 2010 sind in der Schweiz 75 Herzen transplantiert worden, wovon 30 (40\%) an Patienten mit medizinischer Dringlichkeit zugeteilt wurden. Die 245 Leberempfänger im gleichen Zeitraum hatten im Mittel einen MELD-Score von 25, davon hatten jedoch 113 (46\%) Empfänger zwischen 26 und 40 MELD-Punkten. Dies zeigt, dass Herzen und Lebern beinahe zur Hälfte schwer kranken Patienten zugeteilt werden.

Diese Tatsache hat bei Herztransplantationen zur Folge, dass sich die Outcomes der Transplantationen tendenziell verschlechtern und die Mortalität zunimmt, was beispielsweise bei Patienten mit einem Kunstherzen (bridge to transplant) der Fall ist [1]. Dies trifft auf Lebertransplantationen nicht in gleichem Masse zu, da umstritten ist, inwiefern der MELDScore als Indikator für die Outcomes der Transplantationen herangezogen werden kann [2, 3]. Allerdings kristallisiert sich heraus, dass es bei MELD-Scores unter 25 keine eindeutige Korrelation zu den Outcomes der Transplantationen gibt, die Resultate der Transplantationen sich jedoch bei höheren MELD-Werten (> 25) zunehmend verschlechtern [4]. Der Blick auf die Tatsache, dass in der Schweiz knapp die Hälfte der Leberpatienten mit MELD-Werten von über 25 transplantiert werden, legt die Vermutung nahe, dass der Grundsatz, den kränksten Patienten eine hohe Priorität bei der Organzuteilung beizumessen, die Outcomes der Transplantationen auch hier negativ beeinflusst.

Model for End-stage Liver Disease) ist ein ScoringSystem, das von 6-40 reicht und die Schwere der Lebererkrankung anhand der Wahrscheinlichkeit der 3-Monats-Mortalität angibt.

\section{Ökonomisch}

Zwar ist die Transplantation, verglichen mit anderen Therapieformen in den meisten Fällen und bezogen müssen, als auch die längeren Hospitalisationszeiten bei kränkeren Patienten nach der Transplantation verursachen enorm hohe Ausgaben. Und da sich aufgrund der langen Wartezeit der Gesundheitszustand der initial weniger kranken Patienten verschlechtert, ist nicht nur ihre prä-, sondern wird auch ihre postoperative Behandlung teurer $[5,6]$.

\section{Ethisch}

Die Folgen der Organzuteilung gemäss Transplantationsgesetz stellen den Gesetzgeber auch vor ein moralisches Problem: Aufgrund des Organmangels kommen im Wesentlichen diejenigen Patienten zum Zug, die dringend auf ein Organ angewiesen sind. Derweil haben die übrigen Patienten auf der Warteliste quasi keine andere Wahl, als so lange zu warten, bis sie krank genug sind, um in Übereinstimmung mit dem Gesetz ein Spenderorgan zugeteilt $\mathrm{zu}$ bekommen. Darüber hinaus muss mit der geltenden Regelung in Kauf genommen werden, dass - von Organ zu Organ in unterschiedlichem Masse - die derart verteilten Spenderorgane bei weitem nicht $\mathrm{zu}$ einem maximalen Nutzen gereichen.

Grundsätzlich stehen wir hinsichtlich der Zuteilung von Spenderorganen vor der Wahl, das Überleben von den am schwersten kranken Patienten so weit möglich zu sichern, oder den Nutzen (verstanden als Lebensqualität und Lebenserwartung) von weniger schwer kranken Patienten zu maximieren. Im ersten Fall wird sich der Zustand von ursprünglich weniger gravierend kranken Patienten durch die Wartezeit so weit verschlechtern, bis ihnen - um ihr Überleben zu sichern - ein Organ zugeteilt wird. Im zweiten Fall kann der Nutzen einiger (der weniger schwer kranken Patienten, bei denen mit einem besseren Outcome zu rechnen ist) nur durch das «Opfer» weniger (der schwer kranken Patienten) maximiert werden.

Dies ist ethisch äusserst unbefriedigend, denn die Wahl eines der erwähnten Prinzipien kann die moralischen Ansprüche, die jeder der betroffenen Patienten hat, nicht auflösen, weil moralische Ansprüche einer Person sich nicht dadurch aufheben lassen, dass eine andere Person in derselben Situation höher gewichtete moralische Ansprüche hat [7]. 


\section{Eine Alternative}

Nichtsdestotrotz muss in Anbetracht der skizzierten medizinischen und ökonomischen Probleme wie auch des moralischen Dilemmas eine Lösung gefunden werden, die alle relevanten Faktoren berücksichtigt. Eine akzeptable Lösung des Zuteilungsproblems muss folgende Bedingungen erfüllen:

1. Verhinderung von Willkür bei der Zuteilung von Spenderorganen

2. Legitimität

3. Berücksichtigung relevanter moralischer Werte

4. das System muss dem permanenten Organmangel Rechnung tragen

Die erste Bedingung wird dadurch erfüllt, dass die $\mathrm{Zu}$ teilungsregeln gesetzlich festgeschrieben werden. Die zweite (Legitimität) wird dadurch garantiert, dass das Allokationssystem der Öffentlichkeit zugänglich und in seinen Grundzügen für sie verständlich ist und Gegenstand öffentlicher Diskussionen und Revisionen sein kann. Die Erfüllung der dritten Bedingung hängt von der Rechtfertigbarkeit der berücksichtigten moralischen Werte, beziehungsweise der Rechtfertigbarkeit, warum andere Werte nicht berücksichtigt werden, ab. Die vierte Bedingung bedeutet, dass das System sowohl medizinisch wie auch ökonomisch gewissen Effizienzkriterien gerecht werden muss.

Die grösste Schwierigkeit dürfte zunächst in der Wahl ethischer Zuteilungsprinzipien bestehen, die dem permanenten Organmangel Rechnung tragen. Vor dem Hintergrund der Erfahrung der aktuellen Zuteilungspraxis im Verbund mit theoretischen Überlegungen wird schnell klar, dass es eine Reihe von Verteilungskriterien gibt, die zwar plausibel, jedoch nicht hinreichend sind, da jedes für sich allein genommen nicht alle relevanten moralischen Werte zu berücksichtigen vermag. Erst ein Set von adäquat ge- wichteten Prinzipien kann diesem Anspruch gerecht werden. Dieser Ansatz erlaubt die Wahl von gutbegründeten Verteilungskriterien, die dank der Berücksichtigung vielfältiger moralischer Intuitionen ein von allen tragbares Verteilungsverfahren ermöglicht [7].

Persad, Wertheimer und Emanuel [8] befürworten ein alternatives System, das mehrere moralisch relevante Zuteilungsprinzipien kombiniert, und sie nennen das daraus resultierende System das complete lives system, da es ganze Leben anstatt nur Ereignisse oder Episoden einzelner Leben in Betracht zieht. Unter anderem werden von den Autoren folgende zwei Prinzipien als relevant erachtet:

a) Prognose geretteter Lebensjahre

b) Anzahl geretteter Leben.

Diese Auflistung gibt gleichzeitig die Hierarchie der zwei Grundprinzipien, die bei der Verteilung von Organen Anwendung finden sollen, wieder. Die moralische Relevanz beider Prinzipen für sich allein genommen, liegt auf der Hand und dürfte kaum ein Begründungsproblem darstellen. Vielmehr bedarf die Kombination der Prinzipien und deren Hierarchisierung einer Begründung.

a) ist als Prinzip aufzufassen, das auf eine Nutzenmaximierung abzielt, während b) darauf abzielt, Leben zu retten. Im weitesten Sinne kann a) als Entsprechung zum im Transplantationsgesetz genannten Kriterium des medizinischen Nutzens und b) als Entsprechung der medizinischen Dringlichkeit aufgefasst werden. Die beiden Kriterien werden hier jedoch umgekehrt gewichtet, denn die Verschlechterung des Gesundheitszustandes der Patienten auf der Warteliste ist eine direkte Folge der Bevorzugung der kränksten Patienten in einer Situation, in der Mangel herrscht [9]. Die Frage ist daher nicht nur, wer ein Organ erhalten soll, sondern auch, wann jemand ein

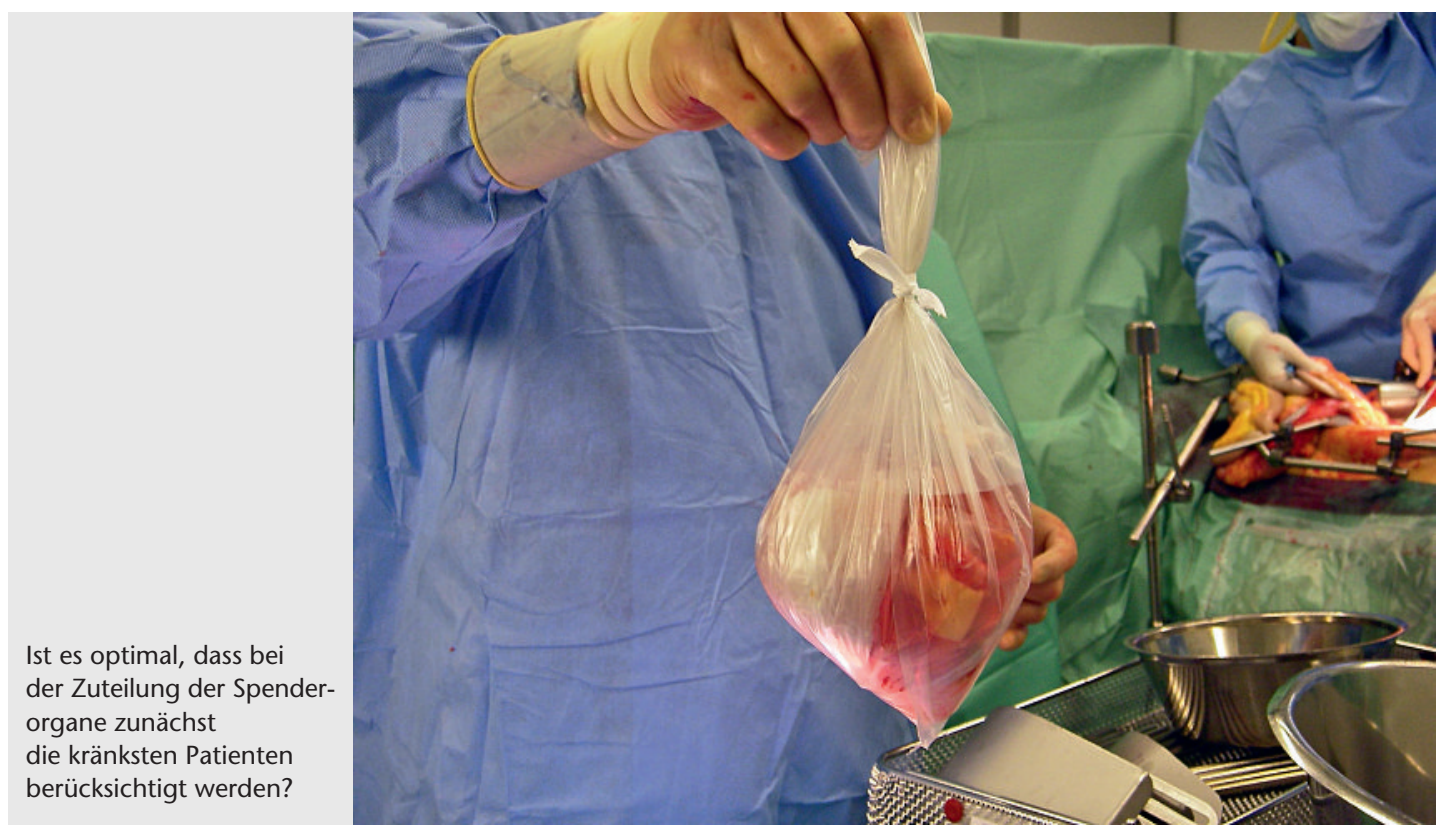


Organ erhalten soll. Im Sinne einer effizienten Verteilung der wenigen zur Verfügung stehenden Organe, sollten Empfänger ein Organ dann erhalten, wenn sie den grössten Gewinn daraus ziehen, was an der Anzahl geretteter Lebensjahre bemessen werden kann. Ein solches Effizienzkriterium würde dann auch der dynamischen Natur des Gesundheitszustandes der Patienten Rechnung tragen.

Wird die Rechtfertigung für das Prinzip a) akzeptiert, so wird damit auch ein weiteres implizites Prinzip anerkannt, das Persad, Wertheimer und Emanuel explizit nennen. Nämlich, dass jüngere Patienten Priorität vor älteren haben. Denn je jünger ein Patient, desto mehr Lebensjahre können (potentiell) gerettet werden. Der Vorwurf, damit ältere Patienten zu diskriminieren, wird durch den Einwand entkräftet, dass jeder, der 65 Jahre alt ist, einmal 25 Jahre alt gewesen ist [8].

\section{Die Gewichtung der Priorität bei der Zuteilung von Organen sollte zugunsten des medizinischen Nutzens verschoben werden}

Zuteilung von Organen zugunsten des medizinischen Nutzens zu verschieben. Die vorangehenden Erörterungen haben gezeigt, dass es gute Gründe gibt, in erster Linie die Anzahl geretteter Lebensjahre als Massstab für eine gerechte Zuteilung heranzuziehen. Damit werden auch medizinisch dringliche Patienten nicht per se von der Verteilung ausgeschlossen, denn ein gerettetes Leben im Verbund mit einer guten Prognose des Resultates einer Transplantation hat in einem solchen System grosses Gewicht.

\section{Literatur}

1 Patlolla V, Patten RD, Denofrio D, Konstam MA, Krishnamani R. The effect of ventricular assist devices on post-transplant mortality - an analysis of the united network for organ sharing thoracic registry. J Am Coll Cardiol. 2009;53:264-71.

2 Habib S, Berk B, Chang CCH, Demetris AJ, Fontes P, Dvorchik I et al. Meld and prediction of post-liver transplantation survival. Liver Transpl. 2006;12: 440-7.

3 Onaca NN, Levy MF, Netto GJ, Thomas MJ, Sanchez EQ, Chinnakotla S et al. Pretransplant MELD score as a predictor of outcome after liver transplantation for chronic hepatitis c. Am J Transplant. 2003;3:626-30.

4 Desai NM, Mange KC, Crawford MD, Abt PL, Frank AM, Markmann JW et al. Predicting outcome after liver transplantation: utility of the model for end-stage liver disease and a newly derived discrimination function. Transplantation. 2004;77:19-21.

Trotz der prima facie korrekt erscheinenden Intention des Gesetzgebers, medizinisch dringliche Patienten bei der Zuteilung von Organen prioritär zu behandeln, zeigt sich auf den zweiten Blick, dass dieser Grundsatz in der Praxis nicht ohne Probleme bleibt. So ergeben sich auf der einen Seite sowohl medizinisch wie auch ökonomisch Konsequenzen, die sich nicht mit dem Verweis auf die grundsätzliche Legitimität des Prinzips ignoriert werden können. Schliesslich müssen die Konsequenzen von der ganzen Gesellschaft getragen werden, was der Tatsache geschuldet ist, dass solche Probleme der Verteilungsgerechtigkeit zunehmend auch an ökonomische Kontexte gebunden sind. Und nicht zuletzt ist es mehr als fraglich, ob ein derartiger Umgang mit einem solch raren Gut ethisch vertretbar ist.

Eine mögliche Herangehensweise an das Problem besteht darin, die Gewichtung der Priorität bei der
5 Washburn WK, Pollock BH, Nichols L, Speeg KV, Halff G. Impact of recipient MELD Score on resource utilization. Am J Transplant. 2006;6:2449-54.

6 Earl T, Cooil B, Rubin J, Chari R. Cost prediction in liver transplantation using pretransplant donor and recipient characteristics. Transplantation. 2008;86:238-44.

7 Sellmaier S, Vossenkuhl W. Moralische Ansprüche von Patienten und die Allokation von Spenderorganen. In: Oduncu FS, Schroth U, Vossenkuhl W (Hrsg.). Transplantation, Organgewinnung und -allokation. Göttingen: Vandenhoeck und Ruprecht; 2003. S. $131-145$

8 Persad G, Wertheimer A, Emanuel EJ. Principles for allocation of scarce medical interventions. Lancet. 2009;373:423-31.

9 Howard DH. Hope versus efficiency in organ allocation. Transplantation. 2001;72:1169-73. 